\title{
SÍNDROME DA MORTE SÚBITA NA INFÂNCIA*
}

\author{
SUDDEN INFANT DEATH SYNDROME
}

Eumênia C. C. Castro ${ }^{1} \&$ Luiz C. Peres ${ }^{2}$

\begin{abstract}
'Docente da Disciplina de Patologia Geral da Faculdade de Medicina do Triângulo Mineiro. ${ }^{2}$ Docente do Departamento de Patologia da Faculdade de Medicina de Ribeirão Preto da Universidade de São Paulo. Trabalho realizado no Departamento de Patologia da Faculdade de Medicina de Ribeirão Preto da Universidade de São Paulo.

CorRespondÊncia: Prof:Dr. Luiz Cesar Peres. Departamento de Patologia, Faculdade de Medicina de Ribeirão Preto da Universidade de São Paulo, Ribeirão Preto - SP, 14049-900, Fone: 016-602-3072, Fax: 016633 1068, e-mail: Icperes@fmrp.usp.br.
\end{abstract}

CASTRO ECC \& PERES LC. Síndrome da morte súbita na infância. Medicina, Ribeirão Preto, 31: 584-594, out./dez. 1998.

RESUMO: A Síndrome da Morte Súbita da Infância (SIDS) é uma entidade ainda pouco diagnosticada em nosso meio, mas que se constitui na principal causa de morte no primeiro ano de vida, nos países industrializados. Acomete crianças aparentemente hígidas, bem nutridas e cuidadas, no primeiro ano de vida, porém com nítido pico entre o terceiro $\left(3^{\circ}\right)$ e o quarto (4ํ) mês de vida. Nesta revisão, procuramos mostrar a evolução histórica desta síndrome, bem como analisar as principais hipóteses e fatores de risco ligados a ela, além de mostrar o panorama atual no Brasil e no mundo.

UNITERMOS: Morte Infantil Súbita. Mortalidade Infantil.

\section{INTRODUÇÃO}

\subsection{Definição}

A morte súbita, sob o ponto de vista médico, é sinônimo de morte inesperada, repentina, instantânea, não sendo precedida de verdadeira agonia ${ }^{(1)}$. Do ponto de vista da Medicina Legal, no entanto, a morte súbita é considerada como "a morte que sobrevém mais ou menos rapidamente, em alguns segundos, algumas horas ou até mesmo em alguns dias, mas, de maneira imprevista, atingindo, sem causa aparente, a um indivíduo até então com boa saúde, ou que tenha apresentado apenas ligeiras perturbações, ou, ao menos, tidas como tais pelos que o rodeavam"(1). Tal conceito é aceito até hoje, no país, pelos especialistas em morte súbita.
A morte súbita pode ser vista em qualquer idade, quando simplesmente nos referimos ao tempo de evolução do problema que levou à morte. Esta pode ser esperada, quando ocorre em paciente portador de algum problema que possa justificá-la, ou então inesperada, em caso contrário. Esta última poderá ser explicada pela necropsia ou outras investigações post-mortem ou continuar inexplicada, apesar de todos os estudos. Na Conferência sobre Causa de Morte Súbita na Infância, realizada em Seattle, Washington, em 1963, a morte inesperada e inexplicada na infância foi definida como aquela que ocorreria em uma criança previamente hígida ou na qual os sintomas apresentados antes do evento terminal, cuja duração não deveria exceder quarenta e oito (48) horas, não indicassem esta probabilidade ${ }^{(2)}$.

\footnotetext{
* Monografia apresentada pela médica, Eumênia Costa Cunha Castro, como parte do Exame Geral de Qualificação para a obtenção do grau de Mestre em Patologia Humana, na Faculdade de Medicina de Ribeirão Preto, USP, em junho de 1997.
} 
A Síndrome da Morte Súbita da Infância, SIDS, do inglês "Sudden Infant Death Syndrome", foi reconhecida como entidade própria e definida em um painel de especialistas sob os auspícios do National Institute of Child Health and Human Development, em Bethesda, 1969, como sendo "A morte inesperada de um lactente ou criança de menos de 1 ano, totalmente inesperada pela história e a qual um extenso exame post-mortem é incapaz de indicar uma causa adequada que a justifique" (3), excluindo, portanto, todas aquelas mortes que, mesmo ocorrendo de forma súbita e inesperada, pudessem ter a sua causa, natural ou não, identificada. Desta forma, não apenas o tempo, mas, principalmente, as circunstâncias da morte passaram a ser fundamentais. Posteriormente, a definição foi ampliada para "A morte inesperada de um lactente ou criança de menos de 1 ano, que permanece inexplicada após um completo exame post-mortem, incluindo uma investigação da cena da morte e revisão da história. Casos que não possam se enquadrar nestes padrões, incluindo aqueles sem investigação post-mortem, não devem ser diagnosticados como SIDS ${ }^{\prime(4,5) .}$

\subsection{Panorama da SIDS no mundo e no Brasil}

A SIDS é, há muito, um problema de grande importância nos países desenvolvidos, onde representa a principal causa de morte no primeiro ano de vida ${ }^{(6)}$. Somente na Inglaterra e País de Gales mil e quinhentas (1.500) crianças morrem desta síndrome, por ano ${ }^{(7)}$. Segundo ALBERMAN et al. (1994) ${ }^{(8)}$, que analisaram o número de citações das causas de morte em atestados de óbito, no primeiro ano de vida, incluindo aquelas ocorridas no período neonatal, a SIDS foi a quinta causa mais freqüente, precedida por prematuridade, anomalias congênitas, asfixia e infecção.

A SIDS é muito mais facilmente identificada nos países desenvolvidos, porque, neles, a maioria das causas de morte evitáveis, tais como desnutrição, infecções, anomalias congênitas, intoxicações e violência estão muito melhor equacionadas do que nos países em desenvolvimento. No entanto, é bem possível que, mesmo nestes últimos, ela possa ocorrer, embora com grande chance de não ser diagnosticada, imputando-se a morte a problemas muitas vezes triviais, como infecções respiratórias de pouca importância, a eventos agônicos ou mesmo posteriores à morte, como costuma acontecer com a aspiração de conteúdo gástrico ${ }^{(9)}$.
A importância da SIDS, sua elevada frequiência nos países desenvolvidos e a quase inexistência de estudos, em nosso meio, demonstram a necessidade de difusão do seu conhecimento para a determinação de sua ocorrência, fatores de risco associados e possíveis medidas preventivas. Em recente informativo da Sociedade Brasileira de Pediatria, a Dra Magda Lahorgue, preocupada com estas questões, propôs a criação de um registro nacional de SIDS e o apoio à pesquisa nesta área ${ }^{(10)}$. Em vista do que ocorreu nos países desenvolvidos, onde a redução das mortes por causas evitáveis evidenciou a SIDS, é esperado que o mesmo aconteça no Brasil, principalmente nas áreas mais desenvolvidas, o que transforma a SIDS em um futuro problema de saúde pública, cujo primeiro passo é a identificação de sua ocorrência.

Em levantamento de publicações na América Latina sobre SIDS, nos últimos dez anos (base de dados LILACS ), encontramos apenas vinte e nove (29) artigos publicados. Já com a base de dados MEDLINE, no mesmo período de tempo, encontramos mil, duzentas e sessenta e três (1.263) publicações sobre este assunto. A maioria destas últimas realizou-se em países industrializados, o que denota a importância deste assunto naquele meio. Por outro lado, a escassez de publicações a respeito, na América Latina, reflete a desinformação e o desinteresse sobre o assunto, possivelmente por sua menor incidência perante outros problemas de saúde infantil, que podem mascará-la. A situação no Brasil não é diferente, havendo apenas um único artigo brasileiro dentre estes poucos vinte e nove (29) identificados ${ }^{(11)}$. Neste estudo, efetuado por Victora et al. $(1987)^{(11)}$, os autores realizaram investigação dos óbitos de lactentes na região metropolitana de Porto Alegre, durante um ano, chegando à conclusão de que havia setenta e dois (72) casos de morte súbita. Mais recentemente, Peres $(1998)^{(12)}$ demonstrou a existência de dez casos de SIDS dentre as necropsias de crianças de um (1) a doze (12) meses realizadas no Serviço de Verificação de Óbito do Interior, na cidade de Ribeirão Preto. No entanto, não há estudos epidemiológicos em nosso país para sabermos a sua real incidência e importância.

Mudar este panorama requer a melhor divulgação do assunto, orientando-se para que casos suspeitos sejam adequadamente investigados, incluindo necropsia e, possivelmente, criando-se um Registro Nacional, como o defendido por Nunes $(1996)^{(10)}$. 


\section{HISTÓRICO}

\subsection{Desde quando se supõe que exista a SIDS?}

A morte inesperada na infância não é um problema novo. Norvenius $(1995)^{(13)}$, em recente revisão, refere que os primeiros relatos de crianças encontradas mortas no leito datam de 500 A.C. e estão contidos no Primeiro Livro dos Reis, no Velho Testamento. Desde aquela época, as explicações para a morte destas crianças foram interpretadas como sendo conseqüentes ao rolamento de um adulto sobre a criança. Esta interpretação foi tida como correta no decorrer dos séculos, sendo as pessoas que cuidavam das crianças diretamente implicadas na morte, com conseqüências religiosas (excomunhão para os católicos), judiciais e psicológicas muitas vezes devastadoras ${ }^{(13,14,15)}$.

\subsection{SIDS no século XIX}

A visão sobre este problema começou a mudar somente em 1892, quando TEMPLEMAN ${ }^{(16)}$, cirurgião da polícia escocesa, fez observações sobre as características das mortes inesperadas na infância, passando-se a diferenciar a morte súbita das demais causas de morte nesta faixa etária. Este médico relatou o encontro necroscópico de petéquias na superfície dos órgãos torácicos, indicativos de asfixia, e observou que a maioria das mortes ocorria no inverno. Apesar de algumas evidências de que as mortes poderiam ter ocorrido por rolamento de um adulto, ou mesmo por sufocação pela mama ou outras partes do corpo, ele observou que, em diversos casos, não havia qualquer indicação de que tivesse ocorrido qualquer destes problemas e nem mesmo infanticídio, permanecendo estas mortes sem uma adequada explicação da causa que teria motivado a asfixia ${ }^{(16)}$. A partir de então, começaram a surgir diferentes hipóteses para explicá-la.

O encontro de timo volumoso e linfonodos proeminentes, nas necropsias de crianças, levou à chamada teoria do status thymico-lymphaticus, desde as primeiras décadas do século XIX. Acreditava-se que estas estruturas aparentemente volumosas levariam à compressão da traquéia, ocasionando a morte por asfixia. No entanto, diversos autores negaram esta correlação à época ${ }^{(17)}$ e mesmo posteriormente ${ }^{(17,18)}$. BOYD (1932) $)^{(19)}$, demostrou que o timo apresentava crescimento normal, progressivo, desde o nascimento até a puberdade, regredindo posteriormente, e que havia uma interpretação errônea destes órgãos linfóides grandes como sendo patológicos e dos timos involuídos conseqüentes à inanição como sendo os normais. GOLDBLOOM \& WIGLESWORTH $(1938)^{(20)}$ de- monstraram ainda que, em alguns casos de crianças que morreram inesperadamente, foi encontrado um timo de tamanho reduzido, demonstrando, com este artigo, que a compressão da traquéia pelo timo não podia ser implicada como causa de óbito em casos de morte inesperada. Estes autores propuseram, também, que, no exame "post mortem" de crianças vítimas de morte súbita, fossem incluídos estudos bacteriológicos e toxicológicos.

Hoje, sabe-se que o chamado status thymicolymphaticus não passa da condição normal destes órgãos nesta faixa etária. $\mathrm{O}$ timo involui rapidamente na vigência de infecções e outros tipos de estresse, sendo suas alterações histológicas, atualmente utilizadas como parâmetro para determinação do tempo de doença ${ }^{21}$.

\subsection{SIDS no século $X X$}

WERNE \& GARROW (1947) ${ }^{(22)}$ identificaram, em estudo retrospectivo, um aumento de $10 \%$ nos casos de sufocação pelas cobertas, nos anos de 1934 até 1944. Estes autores demonstraram que, na verdade, muitas destas crianças haviam morrido devido a infecções, cardiopatias congênitas e outras patologias, restando apenas um pequeno número de crianças cujas causas de morte permaneceram desconhecidas mesmo após a necropsia.

$\mathrm{Na}$ década de quarenta (40) foi desencadeada uma campanha para prevenção de acidentes, nos Estados Unidos, após ABRAMSON \& YORK (1944) ${ }^{(14)}$ terem demonstrado, em artigo, que havia um número excessivo de mortes por sufocação acidental entre crianças com menos de um (1) ano de idade. Em artigos publicados subsequientemente, demonstrou-se que muitas destas crianças haviam morrido por patologias variadas, tais como cardiopatia congênita, meningite, hemorragia subdural, hidrocefalia e broncopneumonia, entre outras ${ }^{(22)}$. Neste mesmo artigo, os autores demonstraram que, em $74,3 \%$ dos casos em que o exame macroscópico não demonstrava a causa da morte, havia indícios, à microscopia, de que uma doença respiratória fulminante teria sido a sua causa. A partir destes dados, todas as mortes inesperadas, que ocorressem neste período da vida, deveriam ser submetidas à exame post-mortem completo, incluindo necropsia ${ }^{(22)}$.

A análise sistemática das mortes na infância pela necropsia propiciou a identificação de que determinadas mortes inesperadas apresentavam um padrão de sinais diferentes das demais. RABSON \& WAYNE $(1948)^{(23)}$, estudando necropsias de crianças abaixo de dez (10) anos de idade, cujas mortes tinham ocorrido fora do 
ambiente hospitalar, notaram que as mortes inesperadas ocorriam mais em crianças com idade inferior a três (3) anos. No referido estudo, identificaram um grupo com faixa etária entre quatro (4) e oito (8) meses, cujas causas de morte continuavam indeterminadas, mesmo após a autópsia. Em outro trabalho, WERNE \& GARROW (1953) ${ }^{(24)}$ estudaram os achados de necropsia de duzentas e noventa e nove (299) crianças abaixo de um (1) ano de idade, que foram encontradas mortas, as quais estavam em aparente bom estado de saúde antes do óbito. Em 83,2\% destes casos, não havia conclusão sobre a causa de morte, mesmo após a necropsia. STOWENS (1957) ${ }^{(25)}$, em estudo de necropsias de crianças cujas mortes ocorreram de maneira súbita e inesperada, observou que as alterações morfológicas encontradas, em conjunto com as culturas bacterianas negativas, levavam a crer na existência de uma entidade distinta com sinais e sintomas que caracterizariam uma síndrome. Tais crianças estavam na faixa etária de um (1) a cinco (5) meses, com pico de incidência aos três (3) meses. A freqüência de morte inesperada diminuía acima de seis (6) meses de idade, sendo bastante raras acima de um (1) ano. Estes dados foram confirmados por VALDÉSDAPENA $(1958)^{(26)}$, que ainda notou predominância no sexo masculino, em estudo de quarenta e uma (41) crianças achadas mortas no berço.

Finalmente, na Conferência sobre Causa de Morte Súbita na Infânca, em 1963, foi definido que o termo "morte no berço", até então usado corriqueiramente para nomear este problema, era inaceitável para esta entidade, uma vez que algumas destas mortes podiam ocorrer em qualquer local e não apenas nos berços ${ }^{(2)}$. Já nesta época, aventou-se usar o nome "Síndrome da Morte Súbita", o que ocorreu somente em 1969, quando da definição da síndrome ${ }^{(3)}$. O interesse pelo assunto passou a ser intenso, o que pode ser medido pelo número de publicações a partir de então (Figura 1).

\section{EPIDEMIOLOGIA}

\subsection{Faixa etária}

A SIDS ocorre, em geral, a partir da segunda semana de vida, com nítido pico entre o terceiro e quarto mês, não sendo observada após o primeiro ano $^{(27)}$. Esta definição de idade vem sendo modificada, pois alguns autores publicaram estudos, demonstrando a presença de SIDS em neonatos ${ }^{(28,29)}$. A tendência atual, portanto, é considerar como SIDS todas as mortes infantis desde o nascimento que apresentem as características contidas na definição ${ }^{(30)}$. No entanto, deve-se ressaltar que o diagnóstico de SIDS, acima de seis (6) meses de idade e abaixo de um mês de idade, deve ser feito com muito critério e somente após terem sido afastadas todas as demais possibilidades, pois $85 \%$ dos casos ocorrem entre dois (2) e quatro (4) meses e $95 \%$ abaixo dos seis (6) meses ${ }^{(30)}$.

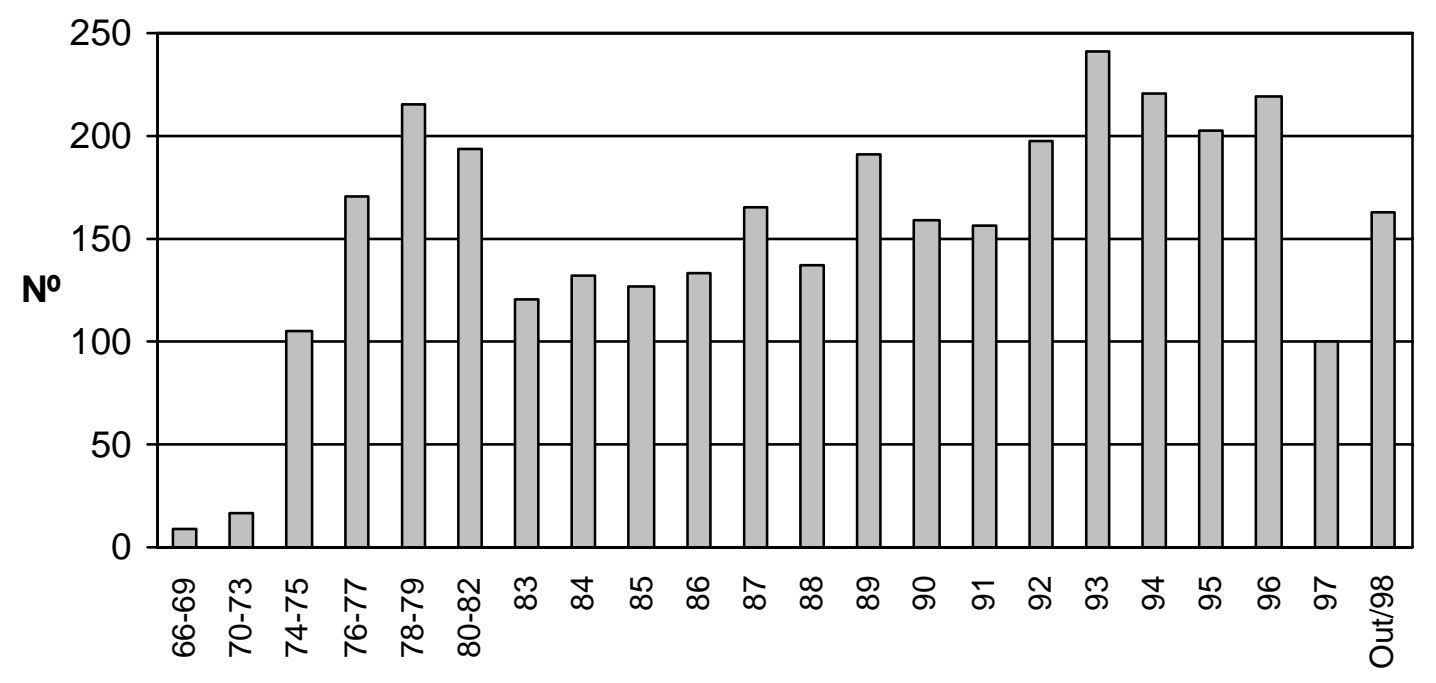

Ano

Figura 1 - Número de publicações de SIDS por ano desde de 1970 (data da publicação da definição). Nota: O número de publicações de 1998 corresponde ao período de janeiro a outubro. 


\subsection{Sexo, raça, recorrência familiar e varia- ção sazonal}

Os meninos são mais afetados do que as meninas e para ambos há maior número de casos no inverno do que no verão. A raça negra parece ser mais suscetível do que a raça branca, seguida pelos hispânicos. Os asiáticos têm a menor taxa de incidência de $\operatorname{SIDS}^{(30)}$.

A taxa de recorrência da SIDS na mesma família, segundo estudos publicados por GUNTHEROTH et al. (1990) $)^{(31)}$, é de treze (13) por mil (1.000) nascidos vivos. IRGENS et al. (1995) ${ }^{(32)}$ apresentam taxa de recorrência de cinco (5) por mil (1.000) nascidos vivos. Em trabalho realizado em Adelaide, Austrália, a taxa de SIDS em gemelares que haviam perdido anteriormente o irmão por SIDS foi de 9,1/1.000 crianças gemelares, nascidas vivas ${ }^{(33)}$.

\subsection{A SIDS no mundo e no Brasil}

A Tabela I mostra a incidência de SIDS por mil crianças nascidas vivas em alguns países. A taxa varia de um país para o outro e também dentro do mesmo país, como os E.U.A., mas são sempre baixas, pois, nestes locais, a mortalidade infantil é sempre menor que 10/1.000 nascidos vivos, muitas vezes abaixo de 5/1.000 nascidos vivos. As diferenças nas taxas refletem inúmeros fatores, desde características genéticas e culturais até fatores sócio-econômicos.

\begin{tabular}{|c|c|}
\hline Local & Incidência \\
\hline \multicolumn{2}{|c|}{ Estados Unidos } \\
\hline - Long Island & $1,46^{(34)}$ \\
\hline - Nebraska & $2,55^{(34)}$ \\
\hline Reino Unido & $2,00^{(34)}$ \\
\hline Finlândia & $0,41^{(35)}$ \\
\hline Israel & $0,67^{(34)}$ \\
\hline Hong-Kong & $0,30^{(36)}$ \\
\hline
\end{tabular}

A Tabela II traz os resultados dos números de casos de SIDS identificados nos dois únicos estudos com metodologia adequada em nosso país. Somente o estudo de Peres $(1998)^{(12)}$, no entanto, foi realizado em um único serviço de necropsia no qual os achados histológicos podiam ser adequadamente analisados.
Estes estudos têm a importância de mostrar que a SIDS existe em nosso meio, faltando apenas identificá-la, baseando-se na definição atual.

\begin{tabular}{|c|c|}
\hline Local & № de casos \\
\hline Porto Alegre & $72 \operatorname{casos}^{(11)}$ \\
\hline Ribeirão Preto & $10 \operatorname{casos}^{(12)}$ \\
\hline
\end{tabular}

\subsection{Fatores de risco}

No momento, o maior avanço observado no estudo da SIDS diz respeito à posição de dormir em decúbito ventral, cujo impacto na redução do número de vítimas tem sido notado em todo o mundo. A relação da posição de dormir com as mortes inesperadas, na infância, vem desde a Idade Antiga, havendo referências no Primeiro Livro dos Reis, no Velho Testamento e, também, na Civilização Helênica, com a citação de Soran de Éfeso sobre a colocação elevada da cabeça dos bebês, ao dormir, cuja interpretação pode ser entendida como orientação para se colocar a criança em decúbito dorsal, segundo Norvenius $(1995)^{(13)}$. No final da década de setenta (70), a hipótese do reflexo oculocardíaco, em que a compressão do olho pelas cobertas poderia levar a uma disfunção cardíaca, levantou suspeitas de que a posição de bruços, ao dormir, poderia ter relação com a morte súbita ${ }^{(37)}$. As evidências iniciais da ligação entre a posição de bruços, ao dormir, e a Síndrome da Morte Súbita na Infância foram feitas na Austrália ${ }^{(38,39)}$. Posteriormente, o grupo de Bristol, região que apresentava a maior incidência de SIDS, no Reino Unido, demonstrou haver forte correlação entre o decúbito ventral e a SIDS, promovendo-se ampla campanha para a mudança da postura, que foi seguida por acentuada redução na incidência $^{(40,41)}$, que passou de 3,7/1.000 nascidos vivos para 0,5/1.000 após a campanha ${ }^{(41)}$. Estudos e programas semelhantes foram feitos em diversos países e regiões com resultados semelhantes, tais como: Noruega $^{(42)}$; Holanda ${ }^{(43)}$ Estados Unidos $^{(44 / 47)}$; Sul da Austrália $^{(48,49)}$; Nova Zelândia ${ }^{(50,51,52)}$; Canada ${ }^{(53)}$ e Tasmânia ${ }^{(54,55)}$.

Muito embora a simples mudança da posição ao dormir se acompanhe de acentuada redução da SIDS, até o momento não existem explicações generalizadamente aceitas sobre os mecanismos da morte. 
Possíveis explicações envolvem a hipertermia ${ }^{(56 / 61)}$, a inalação de gases exalados sob as cobertas ${ }^{(62)}$ e a asfixia por compressão ${ }^{(63)}$. Acredita-se, também, que a criança de bruços tem menor perda de calor pelo dorso e se movimente mais ativamente, sendo, por isso, frequientemente encontrada sob as cobertas, nos pés do berço ${ }^{(58)}$. Recentemente, a obstrução das artérias vertebrais pela rotação e hiperextensão da cabeça, causada pela posição de bruços, foi apontada como possível causa para a Síndrome da Morte Súbita Na Infância $^{(64)}$.

Fatores pós-natais e pré-natais, como idade materna baixa, sexo masculino, tabagismo, aleitamento artificial, abuso do álcool e drogas durante a gestação, status sócio-econômico baixo e outros foram observados como fatores de risco para a SIDS ${ }^{(65,66)}$. O choro, com alta frequência no período neonatal, foi observado em algumas crianças que, posteriormente, morreram de SIDS, sendo, possivelmente, um sinal indicativo de distúrbios neurológicos, potencialmente perigosos ${ }^{(67)}$.

O tabagismo tem sido visto como um dos fatores de risco, de maior importância para a SIDS, a ser evitado. Alguns estudos, nos últimos anos, comprovaram a ligação entre o fumo durante a gestação e SIDS, apesar de ainda não ter sido revelado o seu mecanismo ${ }^{(68 / 78)}$.

Para se minimizarem os riscos de SIDS, diversas medidas têm sido propostas: iniciar o acompanhamento pré-natal o mais precocemente possível; não fumar, válido para ambos os pais ou circunstantes, não fazer uso de álcool ou drogas ilícitas durante a gestação; não colocar as crianças na posição de bruços, ao dormir; não usar colchões de água ou muito moles; não aquecer a criança demasiadamente, tão pouco manter roupas e agasalhos apertados e, por fim, amamentar pelo maior tempo possível ${ }^{(79,80)}$.

\section{HIPÓTESES CAUSAIS}

A procura de fatores etiopatogenéticos para as mortes inesperadas da infância tem sido uma constante ao longo das últimas décadas ${ }^{(81)}$, e sua complexidade pode ser compreendida pelas teorias citadas a seguir: hipersensibilidade ao leite de vaca ${ }^{(82,83)}$; hipersensibilidade a alérgenos domésticos ${ }^{(84)}$; sufocação deliberada ${ }^{(14,22,85)}$; infecção e intoxicação pelo Clostridium botulinum ${ }^{(86)}$; obstrução de vias aéreas superiores $^{(87)}$; traqueobronquite ${ }^{(24)}$; apnéia prolongada do sono $^{(88 / 92)}$; alteração do surfactante pulmonar ${ }^{(7,93,94)}$; problemas cardíacos, tais como defeitos no sistema de condução ${ }^{(95)}$, aumento do intervalo QT durante a pri- meira semana de vida ${ }^{(96)}$, displasia do ventrículo direito ${ }^{(97)}$; defeitos no metabolismo enzimático ${ }^{(30,98)}$; redução do calibre da laringe por hiperplasia das glândulas mucosas subglólitcas ${ }^{(99)}$; alterações do sistema nervoso central e periférico ${ }^{(100)}$, poluição do $\operatorname{ar}^{(101)}$, infecção pelo vírus sincicial respiratório ${ }^{(102)}$; aumento da apoptose neuronal cerebral ${ }^{(103)}$; hipervagotonia familiar ${ }^{(104)}$; distúrbio dos mecanismos envolvidos na postura antigravitacional e intolerância ortostática ${ }^{(105)}$; estímulo superantigênico pela proteína $\mathrm{CPE}$ da cepa $\mathrm{E}$ do Clostridium perfrigens $^{(106)}$; polimorfismo do gene responsável pela codificação das Heat Shock Protein ${ }^{(107,108)}$; aumento da antigenicidade de cepas de E. coli ${ }^{(109)}$, reflexo medo-paralisia ${ }^{(110)}$; deficiência de acetilCoA de cadeia média ${ }^{(111 / 114)}$; outras desordens da $\beta$-oxidação dos ácidos $\operatorname{graxos}^{(114,115)}$; superantígenos ${ }^{(116)}$ e agentes infecciosos ${ }^{(117)}$.

Atualmente, o interesse tem se focalizado na apnéia prolongada de diversas causas ${ }^{(30,58,88 / 92)}$.

Durante os últimos vinte (20) anos, a publicação sobre fatores que poderiam causar a SIDS tem sofrido um aumento considerável. Na década de setenta (70), foram publicados duzentos e noventa e três (293) artigos, envolvendo a etiologia da SIDS; já na década de oitenta (80) foram mil trezentos e cinquenta e nove (1.359), um aumento de 4,6 vezes, mantendo-se elevado na década de noventa (90), com mil, trezentos e sessenta e oito (1.368) artigos. Nos últimos anos, alguns autores têm defendido a posição de que cada caso deveria ser estudado em separado, evitando-se englobar sob o rótulo de SIDS todas estas mortes por causas tão diferentes. Desta forma, o estudo em separado poderia permitir a identificação dos casos inequivocamente devidos à $\operatorname{SIDS}^{(118)}$.

\section{ACHADOS ANÁTOMOPATOLÓGICOS}

Desde há muito tempo, diversos estudiosos da SIDS têm procurado sinais histopatológicos patognomônicos ou, pelo menos, fortemente indicativos desta entidade.

Os achados macro e microscópicos das autópsias de SIDS são, por definição, mínimos ${ }^{(6,30)}$. Petéquias nas superfícies pleural, epicárdica e subcapsulares no timo são os mais comuns, conhecidos desde o século passado ${ }^{(13)}$, decorrentes do aumento da pressão negativa, intratorácica, secundária a uma inspiração vigorosa, indicativa de morte por asfixia ${ }^{(119,120,121)}$. Este aumento da pressão negativa, intratorácica fica evidenciado pelo encontro de petéquias apenas na porção torácica do timo, sendo poupada a cervical ${ }^{(121)}$. 
Estes sinais não são exclusivos da SIDS, podendo mesmo serem observados em fetos com morte intrauterina $^{(30)}$. Hemorragias tímicas, intraparenquimatosas, geralmente, acompanham as petéquias superficiais e têm a mesma conotação ${ }^{(119,121)}$. Nos casos de sufocação mecânica, acidental ou intencional, além destes dados, encontram-se petéquias na face e em conjunti$\operatorname{vas}^{(120)}$. O encontro de petéquias em órgãos extratorácicos decorre de outros mecanismos ${ }^{(122)}$. Grande parte das crianças vítimas de SIDS apresentam edema pulmonar, observado macro e microscopicamente. Acredita-se que este fenômeno esteja relacionado com as alterações agônicas, não sendo conseqüente a problemas cardíacos prévios ${ }^{(119,121)}$.

Alguns estudos demonstraram, ainda, sinais de hipóxia aguda e crônica ${ }^{(123,124,125)}$ em pacientes vítimas de SIDS, demonstrando que o termo morte súbita não se aplica em alguns casos.

O sistema respiratório de crianças vítimas de SIDS foi objeto de diversos estudos para a identificação de mecanismos que justificassem os sinais de asfixia. Entre estes, encontramos os relativos às vias aéreas superiores. VALDÉS-DAPENA (1958) ${ }^{(26)}$ chamou a atenção sobre uma lesão da laringe, mais precisamente das cordas vocais, caracterizada por espessamento da membrana basal do epitélio estratificado, interpretado como necrose fibrinóide. Segundo CULLITY \& EMERY (1974) ${ }^{(126)}$, o espessamento da membrana basal era compatível com colágeno. SHATZ et al. $(1991,1994){ }^{127,128}$ demonstraram maior ocorrência de espessamento da membrana basal das cordas vocais, em pacientes com SIDS, propondo que este sinal fosse utilizado como um marcador desta condição, nas autópsias de crianças vítimas de morte súbita. Recentemente, CASTRO \& PERES ${ }^{(129)}$ demonstraram que este espessamento, constituído por componentes intrínsecos da membrana basal, ocorre em crianças menores de um ano cujas mortes foram devidas a outras causas que não SIDS, não sendo, portanto, exclusivo desta entidade.

\section{PROTOCOLO DE ESTUDO}

O protocolo de estudo da SIDS inclui: necropsia completa, exame do cenário de morte, boa história clínica, estudo toxicológico e do estado imunológico, radiografia do corpo inteiro, estudos metabólicos, culturas de tecido e fluidos para vírus e bactérias e um acesso rápido à criança, logo após a sua morte ${ }^{(130)}$. Quanto à história clínica, EMERY \& CROWLEY
$(1956)^{(131)}$ demonstraram, pela revisão das histórias obtidas com os familiares no dia da morte, que, em somente cinco (5) de cinquenta (50) casos, os relatos tinham o mesmo conteúdo em todas as versões. Em alguns casos, as crianças estavam doentes e a discrepância, nestas histórias, se devia, possivelmente, ao trauma da morte inesperada. Concluiu-se, portanto, que a descrição do cenário da morte por uma outra pessoa que não estivesse envolvida com o caso seria mais fidedigna. Esta investigação é importante para o diagnóstico diferencial entre a SIDS e as mortes acidentais ou infanticídios ${ }^{(132)}$, porém é difícil de ser realizada, quando não impossível, em algumas localidades, como é o caso de nosso país, impossibilitando o diagnóstico desta síndrome com base na definição ampliada $^{(4,5)}$. Por estes motivos, alguns autores acreditam que o estudo do cenário da morte traz o risco potencial de subdiagnóstico ${ }^{(133)}$.

Acreditamos que, no Brasil, se deva, inicialmente, fundamentar o diagnóstico de SIDS com base nos critérios contidos na definição original ${ }^{(3)}$, pois estamos em uma fase em que a necessidade é de se identificar o problema para posteriormente aprofundar os estudos com critérios mais rígidos. Se quisermos introduzir estes últimos de imediato, retardaremos a identificação do problema, com conseqüências desastrosas.

\section{CONCLUSÃO}

A Síndrome da Morte Súbita na Infância é reconhecida como um problema de saúde pública, em diversos países. No Brasil, são identificadas outras condições de maior impacto na saúde pública, como as doenças infecciosas, gastrintestinais e desnutrição, mas, da mesma forma que aconteceu naqueles países, está havendo diminuição na incidência de doenças evitáveis através de campanhas de vacinação, da maior atenção à saúde pública e da melhoria das condições gerais de vida. Desta forma, problemas como a SIDS deverão ser cada vez mais freqüentes em nosso país, juntamente com as anomalias congênitas e doenças neoplásicas da infância. É necessário, portanto, começar um programa de conscientização dos profissionais da área da saúde, bem como da população em geral, para que se identifiquem melhor estes casos, a fim de se permitir o seu estudo e, conseqüentemente, o planejamento das ações necessárias.

O termo SIDS deve ficar restrito aos casos completamente estudados, o que inclui exame postmortem, com necropsia completa, sendo preferível 
definir os casos com características semelhantes, porém incompletamente estudados, como morte indeterminada, para fins de emissão da Declaração de Óbito, com o intuito de se formar uma casuística fidedigna, a fim de dimensionar o problema.
A educação ainda é uma das formas mais eficazes para se conseguir a prevenção de grande parte das doenças, por isto acreditamos que a melhor difusão do conhecimento sobre a SIDS seja absolutamente necessária.

CASTRO ECC \& PERES LC. Sudden infant death syndrome. Medicina, Ribeirão Preto, 31: 584-594, oct./dec. 1998.

ABSTRACT: Sudden Infant Death Syndrome (SIDS) is seldom recognized in our country although it is the leading infant death cause in the first year in industrialized countries. It is seen in apparently healthy, well-nourished and cared for children in the first year of life with a peak incidence between the $3^{\text {rd }}$ and $4^{\text {th }}$ month. The aim of this revision is to show how it has been historically seen, to analyze the most important hypotheses and the risk factors associated to it as well as to show the present situation in Brazil and in the world.

UNITERMS: Sudden Infant Death, Infant Mortality.

\section{REFERÊNCIAS BIBLIOGRÁFICAS}

1 - FÁVERO F. Morte súbita-morte agônica-sobrevivência. In: FÁVERO F, ed. Medicina legal, 8th ed. Livraria Martins Editora, Rio de Janeiro, p.151-153, 1966.

2 - VALDES-DAPENA M. Sudden and unexpected death in infancy: A review of the word literature. Pediatrics 39: 123-137, 1967.

3 - BECKWITH JB \& RAY CG. Sudden infant death syndrome. University of Washington Press 18, Washington, DC, 1970.

4 - ZYLKE JW. Sudden infant death syndrome: resurgent research offers hope. JAMA 262:1565-1566, 1989.

5 - WILLINGER M; JAMES LS \& CATZ C. Defining the Sudden Infant Death Syndrome (SIDS): Deliberations of an expert panel convened by the National Institute of Child Health and Human Development. Pediatr Pathol 11: 677-684, 1991.

6 - VALDES-DAPENAM. Sudden infant death syndrome: Overview of recent research developments from a pediatric pathologist's perspective. Pediatrician 15: 222-230, 1988.

7 - BERRY PJ et al. Surfactant abnormality and the sudden infant death syndrome a primary or secondary phenomenon? Arch Dis Child 65:774-778, 1990.

8 - ALBERMAN E et al. A new hierarchical classification of causes of infant deaths in England and Wales. Arch Dis Child 70: 403-409, 1994.

9 - HIRSCH CS \& ZUMWALT RE. Forensic pathology. In DAMJANOV I \& LINDER J, eds. Anderson's pathology, 10th ed. C.V. Mosby, St Louis, p. 83-85, 1996.

10 - NUNES ML. Síndrome da morte súbita na infância. Este é o momento de iniciarmos um registro nacional. Informativo da Sociedade Brasileira de Patologia, p.3, 1996.

11 - VICTORA CG et al. Quadro epidemiológico das mortes súbitas na infância em cidades gaúchas. Rev Saude Publica 21: 490-496, 1987.
12 - PERES LC. Sudden Infant Death Syndrome in Ribeirão Preto, Brazil. Rev Paul Med, 1998 (Em publicação).

13 - NORVENIUS G. Is SIDS a new phenomenon? Scand Univers Press 2: 11-14, 1995.

14 - ABRAMSON H \& YORK N. Accidental mechanical suffocation in infants. J Pediatr 25: 404-413, 1944.

15 - GUNTHEROTH WG, BREAZEALE D \& MCCOUGH GA. The significance of pulmonary petechiae in crib death. Pediatrics 52: 601-608, 1973.

16 - TEMPLEMAN C. Two-hundred and fifty-eight cases of suffocation of infants. Med Chir Soc (Edimburgh) 6 : 326-328, 1892.

17 - FRIEDLEBEN, 1845 Apud GOLDBLOOM BA \& WIGLESWORTH FW. Sudden death in infancy. Can Med Assoc J 2: 119129, 1938.

18 - YOUNG \& TURNBULL, 1931 Apud BOYD E. The weight of the thymus gland in health and in disease. Am J Dis Child 43:1162-1214, 1932.

19 - BOYD E. The weight of the thymus gland in health and in disease. Am J Dis Child 43:1162-1214, 1932.

20 - GOLDBLOOM BA \& WIGLESWORTH FW. Sudden death in infancy. Can Med Assoc J 2: 119-129, 1938.

21 - VAN BAARLEN J, SCHUURMAN K \& HUBER J. Acute thymus involution in infancy and childhood. A reliable marker for duration of acute illness. Hum Pathol 19:1155-1160, 1988.

22 - WERNE J \& GARROW I. Sudden deaths of infants allegedly due to mechanical suffocation. Am J Public Health 37: 675-687, 1947.

23 - RABSON SM \& WAYNE F. Sudden and unexpected natural death. J Pediatr 20:166-172, 1948.

24 - WERNE J \& GARROW I. Sudden apparently unexplained death during infancy.I Pathologic findings in infants found dead. Am J Pathol 29: 633-675, 1953. 
25 - STOWENS MD. Sudden unexpected death in infancy. J Dis Child 94: 674-680, 1957.

26 - VALDES-DAPENAM. Crib deaths and focal fibrinoid necrosis of the infant larynx. J Forensic Sci 3: 503-509, 1958.

27 - KNIGHT, B. Sudden death in infancy. In: KNIGHT B, ed. Forensic pathology, Edward Arnold, London, p. 414-421, 1991.

28 - POLBERGER S \& SVENNINGSEN NW. Early neonatal sudden infant death and near death of fullterm infants in maternity wards. Acta Paediatr Scand 74: 861-866, 1985.

29 - RODRIGUEZ-ALARCON J et al. Early neonatal sudden death or near death syndrome. An epidemiological study of 29 cases. Acta Paediatr 84: 704-708, 1994.

30 - VALDES-DAPENA M; NAEYE RL \& BARNESS G. Sudden Infant Death Syndrome. In: BARNESS G, ed. Potter's pathology of the fetus and infant, Mosby, St Louis, p. 433-439, 1997.

31 - GUNTHEROTH WG; LOHMANN R \& SPIERS SP. Risk of sudden infant death syndrome in subsequent siblings. J Pediatr 116: 520-524, 1990.

32 - IRGENS LM et al. Sleeping position and sudden death infant syndrome in Norway 1967-91. Arch Dis Child 72: 478-482, 1995.

33 - BEAL S. Sudden infant death syndrome in twins. Pediatrics 84: 1038-1044, 1989.

34 - VALDES-DAPENA M. Sudden infant death syndrome: A review of the medical literature. Pediatrics 66: 597-614, 1980.

35 - RINTAHAKA PJ \& HIRVONEN J. The epidemiology of sudden infant death syndrome in Finland in 1969-1980. Forensic Sci Int 30: 219-233, 1986.

36 - LEE NNY et al. Sudden infant death syndrome in Hong Kong: confirmation of low incidence. BMJ 298: 721, 1989.

37 - HAYES RW \& BREARTY E. An oculo-cardiac hypothesis of sudden unexpected death in infants. Med Hypoteses 5: 477-480, 1979.

38 - BEAL S. Sleeping position and Sudden infant death syndrome. Med J Aust 149: 562, 1988.

39 - BEAL SM. An overview of retrospective case-control studies investigating the relationship between prone sleep position and SIDS. J Paediatr Child Health 27: 344-348, 1991.

40 - FLEMING PJ et al. Interaction between bedding and sleeping position in the sudden infant death syndrome: a population based case-control study. BMJ 301:85-89, 1990.

41 - WIGFIELD RE et al. Can the fall in Avon's sudden infant death syndrome rate be explained by changes in sleeping position? BMJ 304: 282-283, 1992.

42 - MARKESTAD T et al. Sleeping position and sudden infant death syndrome (SIDS): effect of an intervention programme to avoid prone sleeping. Acta Paediatr 84: 375-378, 1995.

43 - ENGELBERTS AC; JONGE GA \& KOSTENSE PJ. An analysis of trends in the incidence of sudden infant death in the Netherlands 1969-1889. J Pediatr Child Health 27: 329-333, 1991.
44 - POTGIETER ST \& KIBEL MA. Sleeping position of infants in Cape Peninsula. S Afrd Med J 81: 355-357, 1992.

45 - DWYER T. Sudden infant death syndrome-insights from epidemiological research. J Epidemiol Community Health 46: 98-102, 1992.

46 - SPIERS PS \& GUNTHEROTH WG. Recommendations to avoid prone sleep position and recent statitics for sudden infant death syndrome in the United States. Arch Pediatr Adolesc Med 148: 141-146, 1994.

47 - BURD L. Prevalence of prone sleep position and selected infant care practises of North Dakota infants: a comparision of withes and Native Americans. Public Health Rep 109: 446-449, 1994.

48 - BEAL SM \& FINCH CF. An overview of retrospective casecontrol studies investigating the relationship between prone sleep position and SIDS. J Paediatr Child Health 27: 334-339, 1991

49 - BEAL SM. Sudden Infant death syndrome (SIDS) in South Australia related to sleeping conditions. Med J Aust 158: 723, 1993.

50 - HASSALL IB \& VANDENBERG M. Infant sleep position: a New Zeland survey. N Z Med J 98: 97-99, 1985.

51 - DAVIDSON-RADA J; CALDIS S \& TONKIN SL. New Zealand's SIDS prevention program and reduction in infant mortality. Health Educ Q 22:162-171,1995.

52 - MITCHELL EA; ALEY P \& EASTWOOD J. The national cot death prevention program in New Zeland. Aust Public Health 16: 158-161, 1992.

53 - WATERS KA et al. Face strait-down and face near-straightdown position in healthy, prone sleeping infants. J Pediatr 128: $616-625,1996$

54 - DWYER T et al. The contribuition of changes in the prevalence of prone sleeping position to the decline in sudden infant death syndrome in Tasmania. JAMA 273: 783-789, 1995.

55 - DWYER T et al. Prone sleeping position and SIDS: evidence from recent case control and cohort studies in Tasmania. $\mathbf{J}$ Pediatr Chid Health 27: 340-343, 1991.

56 - NELSON EA, TAYLOR BJ \& WEATHERALL IL. Sleeping position and infant bedding may predispose to hypertermia and the sudden infant death syndrome. Lancet 28: 199-201, 1989.

57 - HAALAND K \& THORESEN M. Crib detah, sleeping position and temperature. Tidsskr Nor Laegeforen 112: 1466-1470, 1992.

58 - FLEMING PJ et al. Interactions between thermoregulation and the control of respirationship to sudden infant death. Acta Pediatrics: 57-59, 1993. Suppl.

59 - HELWEG-LARSEN K; LUNDEMOSE JB \& BILLE H . Overheating and sudden infant death. Temperature regulation in relation to the prone position, the possible pathogenesis of sudden infant death. Ugeskr Laeger 156: 7193-7196, 1994.

60 - WILLIAMS SM et al. Temperature and the sudden infant death syndrome. Paediatr Perinat Epidemiol 10: 136-149, 1996.

61 - ORIOT D et al. Prone position may increase temperature around the head of the infant. Acta Paediatr 87: 10051007,1998 
62 - KEMP JS. Sudden infant death syndrome: The role of bedding revisited. J Pediatr 12: 946-947, 1996.

63 - SIMSON JR \& BRANTLEY RE. Postural asphhyxia as a cause of death in sudden infant death syndrome. J Forensic Sci 22: 178-187, 1976.

64 - PAMPHLETT R \& MURRAY N. Vulnerability of the infant brain stem to ischemia: a possible cause of sudden infant death syndrome. J Child Neurol 11: 181-184, 1996.

65 - STEELE R \& LANGWORTH JT. The relationship of antenatal and postnatal factors to sudden unexpected death in infancy. Can Med Assoc J 94: 1165-1171, 1966.

66 - HOWARD JH \& HILLMAN S. Epidemiology of the sudden infant death syndrome: maternal, neonatal and postnatal risk factors. Clin Perinatol 19: 717-737, 1992.

67 - CORWIN MJ et al. Newborn acoustic cry characteristics of infants subsequently dying of sudden infant death syndrome. Pediatrics 96: 73-77, 1995.

68 - MACDORMAN MFet al. Sudden infant death syndrome and smoking in the United States and Sweden. Am J Epidemiol 146: 249-257, 1997.

69 - RAJS $J$ et al. Sudden infant death syndrome: postmortem findings of nicotine and cotinine in pericardial fluids of infants in relation to morphological changes and position at death. Pediatr Pathol Lab Med 17: 83-97, 1997.

70 - GOLDING J. Sudden infant death syndrome and parental smoking-a literature review. Paediatr Perinat Epidemiol 11: 67-77, 1997.

71 - OYEN N et al. Maternal smoking, birth weight and gestational age in sudden infant death syndrome (SIDS) babies and their surviving siblings. Paediatr Perinat Epidemiol 11: 84-95, 1997.

72 - CNNATTINGIUS S \& NORDSTROM ML. Maternal smoking and feto-infant mortality: biological pathways and public health significance. Acta Paediatr 85:1400-1402, 1996.

73 - LOGAM S, SPENCER N \& BLACKBURN C. Sudden infant death syndrome. Smoking is part of casual chain. BMJ 313: 1332-1333, 1996.

74 - POTTER A, LUMLEY J \& WATSON L. The "new"risk factors for SIDS: is there an association with the ethnic and place of birth differences in incidence in Vistoria, Australia? Early Hum Dev 45: 119-231, 1996.

75 - SCRAGG R et al. Public health policy on bed sharing and smoking in sudden infant death syndrome. N Z Med J 108: 218-222, 1995.

76 - KLONOFF-COHEN HS et al. The efect of passive smoking and tobacco exposure through breast milk on sudden infant death syndrome. JAMA 273: 795-798, 1995.

77 - MOLLER LF. Smoking and sudden infant death. Ugeskr Laeger 156: 7197-7199, 1994.

78 - SCRAGG R et al. Bed Sharing, smoking and alcohol in the sudden infant death syndrome. New Zeland Cot Death Study Group. BMJ 307: 1312-1318, 1993.

79 - FLEMING PJ. Understanding and preventing sudden infant death syndrome. Curr Opinion Pediatr 6: 158-162, 1994.
80 - FLEMING PJ et al. Enviroment of infants during sleep and risk of the sudden infant death syndrome: results of 1993-5 case control study for confidential inquiry into stilbirths and deaths in infancy. Confidential Enquiry Stillbirths and Deaths Regional Coordinators and Researchers. BMJ 313:191-195, 1996.

81 - EMERY JL. O diagnóstico da Síndrome da Morte Súbita do Lactente (SMLS)- O conceito de causa suficiente de morte e identificação de mortes de lactentes de etiologia multifatorial. An Nestlé 49: 8-15, 1994.

82 - PARISH WE et al. Hipersensitivity to milk and sudden death in infancy. Lancet II:1106-1110, 1960.

83 - MISCHANDANI HG; MIRCHANDANI IH \& HOUSE D. Sudden infant death syndrome: Measurement of total and specific serum immunoglobulin E (IgE). J Forensic Sci 29: 425-429, 1983.

84 - TURNER KJ; BALDO BA \& HILTON JMN. Ige antibodies to dermatophagoides pteronyssinus (House-dust mite), Aspergillus fumigatus, and $\beta$ - Lactoglobulin in sudden infant death syndrome. BMJ 1: 357-360, 1975.

85 - EMERY JL; GILBERT EF \& ZUGIBE F. Three crib deaths, a babyminder and probable infanticide. Med Sci Law 28: 205211, 1988.

86 - ARNON SS et al. Intestinal infection and toxin production by clostridium botulinum as one cause of sudden infant death syndrome. Lancet I:1273-1291, 1978.

87 - KELLY DH \& SHANNON DC. Episodic complete airway obstruction in infants. Pediatrics 67: 823-827,1981.

88 - STEINSCHNEIDER A. Prolonged apnea and the sudden infant death syndrome: Clinical and laboratory observations. Pediatrics 50: 646-654, 1972.

89 - SHANNON DC; KELLY DH \& O'CONNELLK. Abnormal regulation of ventilation in infants at risk for sudden-infant-death syndrome. N Engl J Med 297: 747-750, 1977.

90 - WARD SLD et al. Sudden infant death syndrome in infants evaluated by apnea programs in California. Pediatrics 77: 451-457, 1986.

91 - SCHECHTMAN VL et al. Sleep apnea in infants who succumb to the sudden infant death syndrome. Pediatrics 87 : 841-846, 1991.

92 - SCHECHTMAN VL et al. Sleep state organization in normal infants and victims of the sudden infant death syndrome. Pediatrics 89: 865-869, 1992.

93 - HILLS BA, MASTERS IB \& VANCE JC. A novel role for surfactant in the lung with implications for the sudden infant death syndrome. Med Hypotheses 44: 431-434, 1995.

94 - MASTERS IB, VANCE J \& HILLS BA. Surfactant abnormalities in ALTE and SIDS. Arch Dis Child 71: 501-505, 1994.

95 - FU C et al. The immunocytochemical demonstration of a relative lack of nerve fibres in the atrioventricular node and bundle of his in the sudden infant death syndrome (SIDS). Forensic Sci Int 66:175-185, 1994.

96 - SCHWARTZ PJ et al. Prolongation of the QT interval and the sudden infant death syndrome. N Engl J Med 338:17091714, 1998. 
97 - PAWEL BR et al. Sudden death in childhood due to right ventricular dysplasia: Report of two cases. Pediatr Pathol 14: 987-995, 1994.

98 - HOWAT $\mathrm{J}$ et al. Defects of metabolism of fatty acids in the sudden infant death syndrome. BMJ 290: 1771-1773, 1985.

99 - HARRISON DFN. Laryngeal morphology in sudden unexpected death in infants. J Laryngol Otol 105: 646-650, 1991.

100 - KINNEY HC; FILIANO JJ \& HARPER RM. The neuropathology of the sudden infant death syndrome. A review. J Neuropathol Exp Neurol 51: 115-126, 1992.

101 - KNOBEL HH; CHEN CJ \& LIANG KY. Sudden infant death syndrome in relation to weather and opttimetrically measured air polution in Taiwan. Pediatrics 96: 1106-1110, 1995.

102 - LINDGREN C et al. Respiratory syncytial virus infection enhances the response to laryngeal chemostimulation and inhibits arousal from sleep in young lambs. Acta Pediatr 85: 789-797, 1996.

103 - SPARKS DL et al. Increased ALZ-50 immunoreactivity in sudden infant death syndrome. J Child Neurol 11: 101-107, 1996.

104 - LUCET V et al. Is familial hypervagotonia a risk factor for sudden infant death? Arch Pediatr 3: 287-288, 1996.

105 - REID GM \& TERVIT H. Sudden infant death syndrome: orthostatic intolerance and disordered antigravity postural mechanisms. Med Hypoteses 46: 162, 1996.

106 - LINDSAY JA. Clostridium perfrigens type A enterotoxin (CPE): more than just explosive diarrhea. Crit Rev Microbiol 22: 257-277, 1996.

107 - GOZAL D. Deficient heat shock protein expression: a pontential mechanism for the sudden infant death syndrome. Med Hypoteses 46: 52-54, 1996.

108 - RAHIM RA et al. Human heat shock protein gene polymorphisms and sudden infant death syndrome. Arch Dis Child 75: 451-452, 1996.

109 - BETTELHEIM KA et al. Virulence factors associated with strains of Echerichia coli from cases of sudden infant death syndrome . Comp Immunol Microbiol Infect Dis 18: 179-188, 1995.

110 - KAADA B. Fear Paralysis-a possible cause of crib death. Tidsskr Nor Laegeforen 10: 848-852, 1995.

111 - GREGERSEN N et al. Prenatal diagnosis of medium chain acyl CoA dehydrogenase (MCAD) deficiency in a family with a previous fatal case of sudden unexpected death in childhood. Prenat Diagn 15: 82-86, 1995.

112 - OPDAL SH et al. Is the medium chain acyl CoA dehydrogenase G985 mutation involved in sudden infant death in Norway? Eur J Pediatr 154: 166-167, 1995.

113 - BRACKETT JC et al. A novel mutation in medium chain acyl CoA dehydrogenase causes sudden neonatal death. J Clin Invest 94: 1477-1483, 1994.

114 - BENNET MJ \& POWEL S. Metabolic disease and sudden, unexpected death in infancy. Hum Pathol 25: 742-746, 1994.

115 - HARPEY JP; CHARPENTIER C \& PATURNEAU-JOUAS M. Sudden infant death syndrome and inherited disorders of fatty acid beta-oxidation. Biol Neonate 58: 70-80, 1990.
116 - LINDSAY JA et al. Can superantigens triguer sudden infant death? Med Hypoteses 43: 81-85, 1994.

117 - BLACKWELL CC et al. The role of infection agents in sudden infant death syndrome. FEMS Immunol Med Microbiol 9: 91-100, 1994.

118 - GILBERT EF \& BARNESS LA. Sudden infant death syndrome. Is it a cause of death? Arch Pathol Lab Med 117:1246-1248, 1993.

119 - LEE JKL et al. Intrathoracic petechial hemorrages in sudden infant death syndrome. Pediatr Pathol 17: 703, 1997.

120 - NIXON JW et al. Suffocation, choking, and strangulation in childhood in England and Wales: epidemiology and prevention. Arch Dis Child 72:6-10, 1995.

121 - BERRY PJ. Pathological findings in SIDS. J Clin Pathol 45: $11-16,1992$

122 - KROUS HF \& JORDAN J. A necropsy study of distribution of petechiae in non-sudden infant death syndrome. Arch Pathol Lab Med 108: 75-76, 1984.

123 - NAEYE RL. Brain-stem and adrenal abnormalities in the Sudden-infant-death Syndrome. Am J Clin Pathol 66: 526530,1976 .

124 - NAEYE RL. Pulmonary arterial abnormalities in the sudden-infant-death syndrome. N Engl J Med 289: 11671170, 1973.

125 - WILLIAMS A; VAWTER G \& REID L. Increased muscularity of the pulmonary circulation in victims of sudden infant death syndrome. Pediatrics 63: 18-23, 1979.

126 - CULLITY GJ \& EMERY JL. Ulceration and necrosis of vocal cords in hospital and unexpected child deaths. J Pathol 115: 27-31, 1974.

127 - SHATZ A, HISS J \& ARENSBURG B. Basement-membrane thickening of the vocal cords in sudden infant death syndrome. Laryngoscope 101: 484-486, 1991.

128 - SHATZ A et al. Age-related basement membrane thickening of the vocal cords in sudden infant death syndrome (SIDS). Laryngoscope 104: 865-868, 1994.

129 - CASTRO ECC \& PERES LC. Vocal cord basement membrane in non SIDS cases. Ped Develop Pathol, (Em publicação).

130 - WIGGLESWORTH FW et al. Technical method pathological investigations in cases of sudden infant death. $\mathbf{J}$ Clin Pathol 40: 1481-1483, 1987.

131 - EMERY JL \& CROWLEY EM. Clinical histories of infants reported to coroner as cases of sudden unexpected death. BMJ II:1518-1521, 1956.

132 - VALDES-DAPENAM. The sudden infant death syndrome: Pathologic findings. Clin Perinatol 19: 701-716, 1992.

133 - GUNTHEROTH WG; SPIERS PS \& NAEYE RL. Redefinition of the Sudden infant death syndrome: The disadvantages. Pediatr Pathol 14: 127-132, 1994.

Recebido para publicação em 01/04/98

Aprovado para publicação em 10/11/98 